\title{
Fractographic Evaluation of Crack Initiation and Growth in Al-CNTs Nanocomposite Fabricated by Induction Melting
}

\author{
M. MANSOOR* AND M. Shahid
}

School of Chemical and Materials Engineering, National University of Sciences and Technology, Islamabad, Pakistan

\begin{abstract}
Presence of reinforcement material in the matrix plays a vital role in modifying the fracture topography besides mechanisms of deformation and strengthening of a composite. In the present study, Al-CNT composite was prepared using induction melting technique. The composite was cast into copper mold and cold rolled to $\approx 95 \%$ reduction in thickness. In as rolled condition, the composite exhibited a simultaneous increase in yield strength $(>70 \%)$, tensile strength $(>50 \%)$ and hardness $(>40 \%)$. The fracture surfaces of the broken tensile specimens were studied using scanning electron microscope. It was observed that the crack initiation was occurred in the matrix followed by void link-ups for its growth. Micro cracks initiation in the matrix played a vital role to sustain the ductility of the material.
\end{abstract}

DOI: 10.12693/APhysPolA.128.B-276

PACS: $81.05 . \mathrm{Ni}$

\section{Introduction}

The recent trends to strengthen the matrix using filler materials are rapidly transforming from macro- or microscale fillers to nano-scale fillers. Carbon nanotubes (CNTs), being one of the candidate materials, hold a strong promise to augment not only localized but also global physical and mechanical properties of the composite. During last decade, numerous researchers published their work encompassing amelioration in mechanical and physical properties of CNT-Aluminum composites [1], however the mechanism of crack initiation and growth in Al-CNT composite materials was the least addressed area. In present work, the initiation and growth of the cracks in Al-CNT composite were studied by SEM supported fractographic investigations.

\section{Experimental}

The multiwalled carbon nanotubes (MWCNTs) of 10 $\mathrm{nm}$ diameter were used for the present work, which were synthesized using chemical vapor deposition [2]. Aluminum (grade-1199) was used for the matrix material, which was melted in an air induction furnace under multifunctional fluxes. When $\mathrm{Al}$ became semi molten, the nanotubes $(0.2$ vol.\%) were added and heating was further continued for 10 minutes. Afterwards, the melt was cast into copper mold and cold rolled to $0.4 \mathrm{~mm}$ thin strips. Detailed fabrication of the composite is discussed elsewhere [3]

The etched structure of the specimen was studied using an SEM Hardness and tensile tests were carried out in accordance with ASTM E384 and ASTM E8, respectively.

\footnotetext{
* corresponding author; e-mail: muhammadmansoor@scme.nust. edu.pk
}

To evaluate fracture initiation and propagation SEM and energy dispersion spectroscopy (EDS) were used for fractographic studies and area analyses of the, respectively.

\section{Results and discussion}

SEM micrographs of the etched specimens are shown in Fig. 1. In pure aluminum specimen, coarse grains were seen, which were elongated in the rolling direction (Fig 1a). A clear grain boundary was evitable between the grains. However, in the composite specimen no evitable grain boundaries were seen. In scattered regions of the specimen nanotubes aligned in the rolling direction were observed (Fig. 1b). EDS point analysis of the features ascertained the presence of the nanotubes (Fig. 1c). No segregation or clustering of the nanotubes was found in the investigated specimens, which depicted towards their appreciable dispersion in the matrix.

The experimental engineering stress-strain curves of pure aluminum and $\mathrm{Al}$-CNTs composite are shown in Fig. 2. Results of tensile testing showed a substantial increase in yield and tensile strengths of the composite as compare to pure aluminum. An increase more than $70 \%$ and $50 \%$ was observed in yield strength and tensile strength, respectively. Similarly, the hardness of the composite was increased $>40 \%$. However, a slight decrease in the ductility of the composite was observed. The decrease in ductility is related with strengthening effect of the nanotubes in the matrix and associated transition of fracture initiation and growth mechanism, which will be discussed in successive section of fractography. The results of the mechanical testing are tabulated in Table.

SEM micrograph in Fig. 3a shows the fracture surfaces of pure aluminum and $\mathrm{Al} 0.2$ vol.\% MWCNTs. It was observed that pure aluminum demonstrated almost 100\% reduction in area, while in the composite material the reduction in area reduced $(\approx 30 \%)$ after the addition of the nanotubes. 
TABLE

Results of the tensile tests.

\begin{tabular}{c|c|c|c}
\hline \hline & Pure Al & Composite & Change [\%] \\
\hline yield st. [MPa] & $72 \pm 6$ & $124 \pm 3$ & 72 \\
UTS [MPa] & $86 \pm 4$ & $132 \pm 2$ & 53 \\
elongation [\%] & $4.14 \pm 0.25$ & $3.74 \pm 0.45$ & 9.68 \\
hardness [Hv] & $27 \pm 3$ & $39 \pm 4$ & 44
\end{tabular}

Both of the fracture surfaces represented same type of fracture mode i.e. ductile fracture; however, the type of their initiation and propagation was different. Fracture surface of pure aluminum was a typical feature of polycrystalline ductile fracture under tensile loading, where plastic deformation was initiated and propagated by cross-slip. The deformation initiated necking of the specimen, which increased localized tensile stresses. The increased local stresses effectuated rapid plastic deformation and caused rupture of the material. Nearly $100 \%$ reduction in area is a typical feature, where plastic deformation (under cross-slip) continues even after necking, which finally causes the fracture [4].

The fracture surface of the composite specimen was accompanied by considerably decreased reduction in area. At the periphery of the fracture region, a discernable shear lip was also observed (Fig. 3c). Presence of strengthening material(s) could be a cause of the decrease in reduction area. Under loading conditions, micro cracks or voids were generated at or around the strengthening material. Moreover, geometrically necessary dislocations were generated due to the non-compatible strain between the matrix and the strengthening material. There could be three typical conditions in the presence of strengthening material [5]:

- If the strengthening material deformed only elastically; the geometrically necessary dislocations would be located in the matrix.

- If the plastic strains of the matrix and the strengthening material were compatible; a delay in fracture nucleation would be observed.

- If the strengthening material undergone plastic deformation (but was less ductile than matrix); the geometrically necessary dislocations would be divided.

The formation of geometrically necessary dislocations caused internal tensile stresses $\left(\sigma_{a}\right)$, which added up in the applied tensile stresses $\left(\sigma_{b}\right)$ and promoted the formation of micro cracks:

$$
\sigma_{c}=\sigma_{a}+\sigma_{b} .
$$

Micro cracks were formed throughout the volume of the material prior to necking. However, as the matrix was ductile, therefore the tensile strength was less than the stress required for the propagation of micro cracks (Eq. 1) as a result necking occurred prior to the failure. Once necking was initiated, voids link-ups occurred

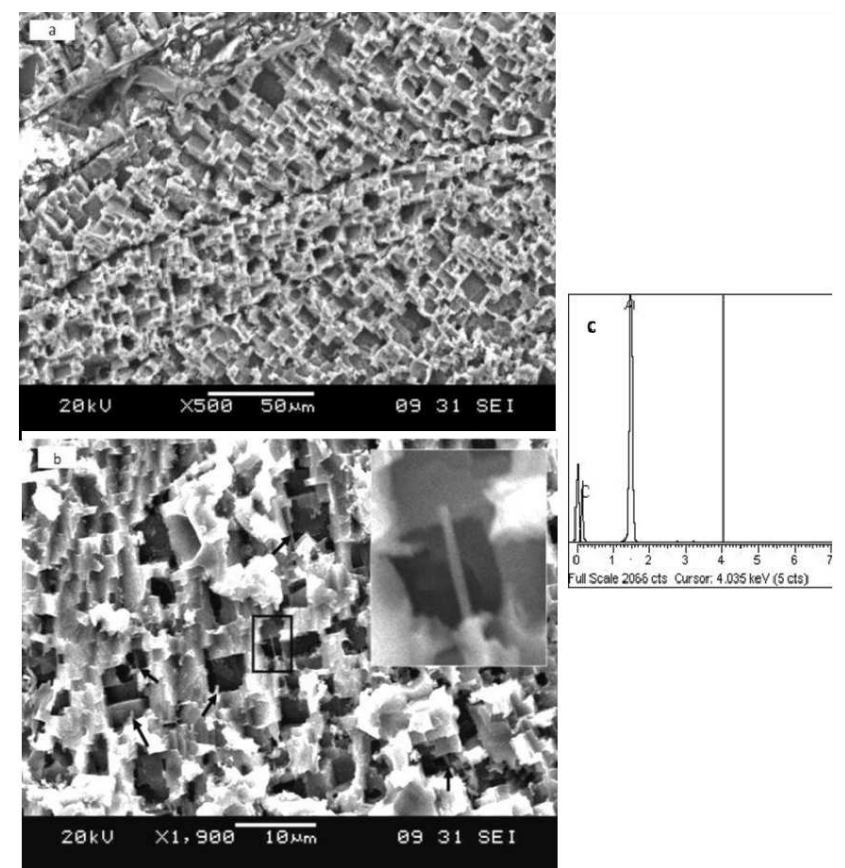

Fig. 1. SEM micrographs of the etched specimens: a) pure aluminum, b) Al-CNTs composite and c) EDS point analysis of the CNTs shown in the inset of $b$ ).

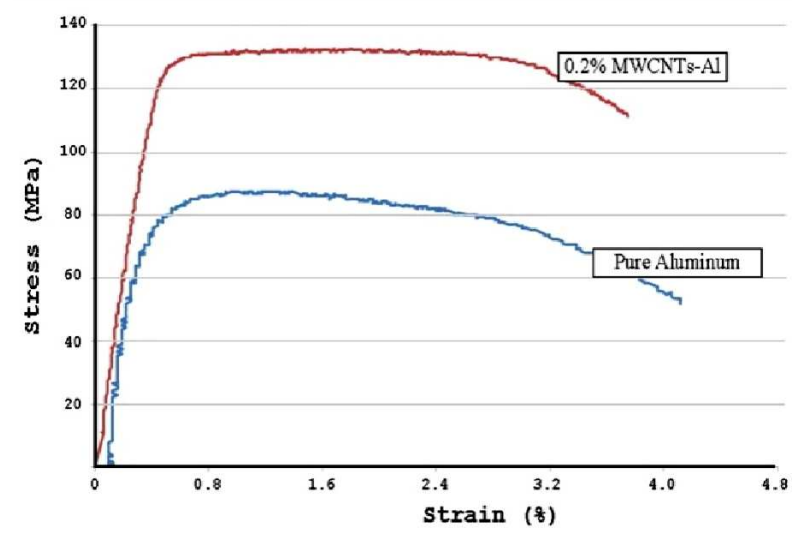

Fig. 2. Experimental engineering stress-strain curves of the pure aluminum and the composite specimens.

in the region because true stress in the necking region was higher than other regions of the specimen. As deformation continued, link-ups of the micro cracks/voids aggravated to a penny shaped crack within the material. The extant of micro cracking depends upon volume fraction, size and yield strength of the strengthening material along with work hardening characteristics of the matrix material. When a big void (penny shaped) was formed, true engineering stress exceeded the tensile strength of the material and final fracture occurred by shearing. Due to the occurrence of shearing fracture, a shear lip surrounded the fracture surface of the composite.

Further, to study the feature and characteristics of the dimples in the fracture surface of the composite higher magnifications were used (Fig. 3b), where uniform dimples were seen with embedded nanotubes in the matrix. 


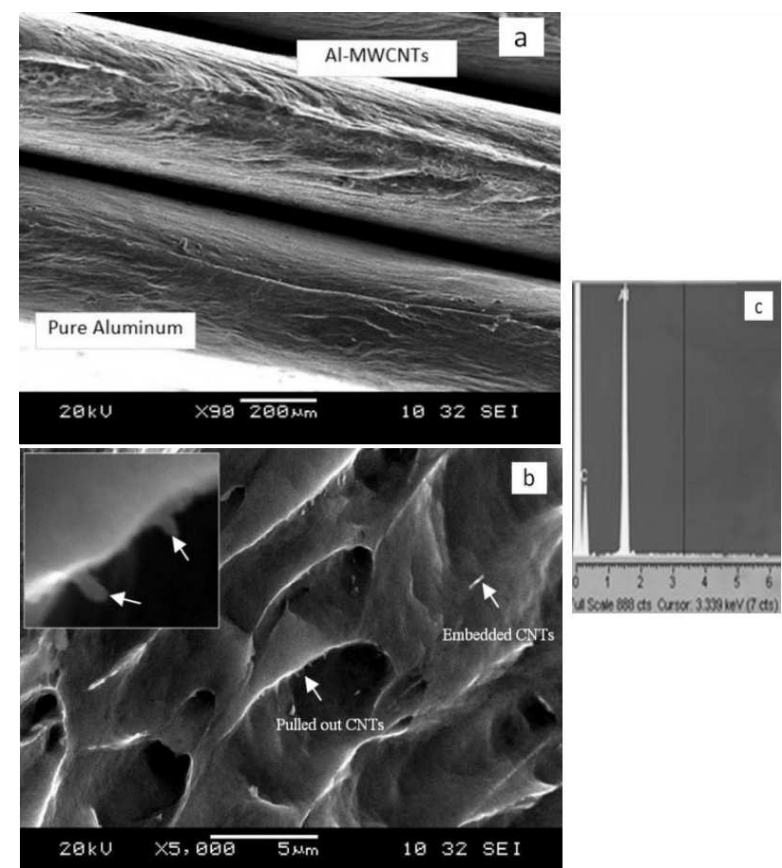

Fig. 3. a) Fracture surface of broken tensile specimens showing reduction in area, b) fracture surface of the composite showing dimples with embedded and pulledout CNTs and c) EDS point analysis of the CNTs present in the matrix.

Presence of the nanotubes was confirmed by EDS point analysis (Fig. 3c). It was also observed that the fracture dimples were hollow and their centers were free from MWCNTs, suggesting that the facture was initiated within the matrix and not from the nanotubes. Additionally, pulled-out nanotubes at the edges of the dimples were seen (inset of Fig. 3b). As the fracture propagated under testing conditions, the nanotubes, which were aligned (due to cold rolling) in the loading direction, were pulled-out by the sliding of the outer shells, contributing towards resistance to the fracture growth.

\subsection{Failure mechanism}

According to the preceding discussion and experimental findings, the mechanism of fracture initiation and propagation in Al-MWCNTs composite could be inferred. It is known that both of the constituent materials of the composite are capable of plastic straining [6], therefore a delayed fracture could be expected. The nanotubes are, additionally, capable of high elastic straining [7]. Therefore, micro crack or voids were initiated in the matrix instead of in the interface of CNT-Al or in the nanotube itself. The fact could be observed in Fig. 3b, where the centers of the dimples were free of any second phase (i.e. MWCNTs). Once initiated, the voids grew in the direction of applied load (i.e. tensile axis). The growth of the voids was driven by plastic deformation of the matrix. Geometrical conditions showed that the strain rate in the voids $\left(\varepsilon_{a}\right)$ was more than overall strain rate $\left(\varepsilon_{b}\right)$ according to the Eq. (2):

$$
\varepsilon_{a}=C \varepsilon_{b}
$$

where $C$ is a constant with value between 1 and 2 . The increased strain rate $\left(\varepsilon_{a}\right)$ caused the final fracture of the composite [5].

\section{Conclusions}

Presence of the nanotubes in aluminum matrix caused a pronounced effect on the fracture initiation and growth mechanism besides mechanical properties. It was inferred that:

- initiation of micro voids/cracks occurred in aluminum matrix instead of nanotubes itself or CNT$\mathrm{Al}$ interface,

- the micro-crack link-ups proceeded within the matrix, therefore pulled-out and embedded nanotubes were present on the lips and body of the dimples, respectively,

- elastic properties of the nanotubes contributed towards retention of the composite ductility such that a feeble loss of the ductility $(<10 \%)$ occurred.

\section{References}

[1] A. Umma, M. Maleque, I. Iskandar, Y.A. Mohammed, Aust. J. Basic Appl. Sci. 6, 69 (2012).

[2] M. Mansoor, M. Shahid, A. Habib, Mat. Res. 17, 739 (2014).

[3] M. Mansoor, M. Shahid, J. Alloy Compd. 643, 74 (2014).

[4] G.E. Dieter, Mechanical Metallurgy, McGraw-Hills, London 1988.

[5] T.H. Courtney, Mechanical Behavior of Materials, 2nd ed., McGraw-Hills, Boston 2000.

[6] P.M. Ajayan, O.Z. Zhou, Carbon Nanotubes, ser. Topics in Applied Physics, vol. 80, Eds.: M.S. Dresselhaus, G. Dresselhaus, P. Avouris, Springer, Berlin 2001.

[7] D. Walter, L. Ericson, M. Casavant, J. Lui, D. Colbert, K. Smith, R.E. Smalley, Appl. Phys. Lett. 74, 3803 (1999) 